\title{
Evaluation of the Nature and Etiologies of Risk Factors for Diaphyseal Atypical Femoral Fractures
}

\author{
Hiroyuki Tsuchie $^{\mathrm{a}}$ Naohisa Miyakoshi ${ }^{\mathrm{a}}$ Yuji Kasukawa ${ }^{\mathrm{a}}$ Koji Nozaka ${ }^{\mathrm{a}}$ \\ Kimio Saito $^{\mathrm{a}, \mathrm{b}}$ Hayato Kinoshita ${ }^{c}$ Moto Kobayashi $^{d}$ Norio Suzuki $^{\mathrm{e}}$ \\ Toshiaki Aizawa $^{f}$ Hidekazu Abe ${ }^{g}$ Shigeto Maekawa ${ }^{\text {h }}$ Takenori Tomite $^{\mathrm{i}}$ \\ Yuichi Ono $^{j}$ Kentaro Ouchik ${ }^{k}$ Nobusuke Shibatal Itsuki Nagahatam \\ Masaaki Takeshiman $^{\mathrm{n}}$ Manabu Akagawa $^{\circ}$ Yusuke Yuasa $^{\mathrm{p}}$ Chie Sato $^{q}$ \\ Yoichi Shimada ${ }^{a}$
}

aDepartment of Orthopedic Surgery, Akita University Graduate School of Medicine, Akita, Japan; ${ }^{b}$ Department of Orthopedic Surgery, Kakunodate General Hospital, Senboku, Japan; 'Department of Orthopedic Surgery, Akita Kousei Medical Center, Akita, Japan; 'Department of Orthopedic Surgery, Hiraka General Hospital, Yokote, Japan; eDepartment of Orthopedic Surgery, Yuri Kumiai General Hospital, Yurihonjyo, Japan; fDepartment of Orthopedic Surgery, Northern Akita Municipal Hospital, Kitaakita, Japan; 9Department of Orthopedic Surgery, Ugo Municipal Hospital, Ugo, Japan; hDepartment of Orthopedic Surgery, Ogachi Central Hospital, Yuzawa, Japan; 'Department of Orthopedic Surgery, Japanese Red Cross Akita Hospital, Akita, Japan; 'Department of Orthopedic Surgery, Nakadori General Hospital, Akita, Japan; kDepartment of Orthopedic Surgery, Yokote Municipal Hospital, Yokote, Japan; 'Department of Orthopedic Surgery, Oga Minato Municipal Hospital, Oga, Japan; mDepartment of Orthopedic Surgery, Omagari Kousei Medical Center, Daisen, Japan; 'Department of Orthopedic Surgery, Honjyo Daiichi Hospital, Yurihonjyo, Japan; ${ }^{\circ}$ Department of Orthopedic Surgery, Akita City Hospital, Akita, Japan; PDepartment of Orthopedic Surgery, Omori Municipal Hospital, Yokote, Japan; 9Department of Orthopedic Surgery, Akita Rosai Hospital, Odate, Japan

\section{Highlights of the Study}

- The nature and etiologies of the risk factors for diaphyseal atypical femoral fractures (AFFs) were investigated.

- Diaphyseal AFFs are affected by bone resorption inhibitor usage, strong femoral curvature, and a serrated change in the femur.

- A low serum 25(OH)D level and serrated change are the risk factors for lateral curvature, and a high serum calcium level is a risk factor for serrated changes.

\section{Keywords}

Atypical femoral fracture $\cdot$ Femoral curvature $\cdot$ Serrated change

\section{Abstract}

Objectives: Differences in mechanisms of subtrochanteric and diaphyseal atypical femoral fractures (AFFs) are speculated in studies that analyzed differences in the patients' karger@karger.com www.karger.com/mpp

Karger $\stackrel{\text { ' }}{5}$ BOPEN ACCESS
(C) 2021 The Author(s)

Published by S. Karger AG, Basel

This is an Open Access article licensed under the Creative Commons Attribution-NonCommercial-4.0 International License (CC BY-NC) (http://www.karger.com/Services/OpenAccessLicense), applicable to the online version of the article only. Usage and distribution for commercial purposes requires written permission.
Correspondence to:

Hiroyuki Tsuchie, tuchikiti@yahoo.co.jp 
background. However, the etiologies of each type of AFF have not been studied in detail. This study aimed to investigate the nature and etiologies of the risk factors for diaphyseal AFFs. Materials and Methods: Eighty consecutive Japanese patients with 91 diaphyseal AFFs (AFF group) and 110 age-matched women with osteoporosis (non-AFF control group) were included. Their clinical data were compared; factors affecting AFFs were investigated, and the etiologies of the risk factors for diaphyseal AFFs were examined. Results: Multivariate analysis revealed that femoral serrated changes, bisphosphonate or denosumab usage, and lateral and anterior femoral curvatures were risk factors for diaphyseal AFFs ( $p<0.0011, p=0.0137$, and $p<0.0001$, respectively). Multivariate analyses revealed that serrated changes and low serum $25(\mathrm{OH})$ D levels affected the lateral curvature $(p=0.0088$ and 0.0205 , respectively), while serrated changes affected the anterior curvature $(p=0.0006)$, each significantly affected the femoral curvature. High serum calcium (Ca) levels, lateral femoral curvature, and anterior femoral curvature were predictors of serrated changes ( $p=0.0146,0.0002$, and 0.0098, respectively). Conclusion: Risk factors for diaphyseal AFFs were bone resorption inhibitor usage, a strong femoral curvature, and serrated changes. Low serum 25(OH)D levels and serrated changes are risk factors for lateral curvature, while a high serum Ca level is a risk factor for serrated changes.

\section{(c) 2021 The Author(s)}

Published by S. Karger AG, Basel

\section{Introduction}

Although bisphosphonates (BPs) are the gold standard for osteoporosis pharmacotherapy, several adverse effects related to their long-term use have been reported due to severely suppressed bone turnover. These include the atypical low-energy subtrochanteric and diaphyseal femoral fractures [1-3], which are typically diagnosed as atypical femoral fractures (AFFs). At 3-5 per 100,000, the incidence of AFFs is low $[4,5]$. However, approximately $30 \%$ of all postmenopausal women have osteoporosis [6]. Therefore, AFFs are an important concern in osteoporosis management, the mainstay of which is the use of bone resorption inhibitors. However, AFFs have also been noted in antiresorptive therapy-naive patients, and multiple etiological factors are considered to be involved in these, including an excessive femoral curvature, low serum 25-hydroxyvitamin D (25(OH)D) levels, diabetes, and glucocorticoid usage [7-13]. Thus, although various factors have been proposed, the etiology remains unclear.

AFFs are, by definition, either subtrochanteric or diaphyseal. A subtrochanteric AFF is a fracture that occurs slightly distal to the lesser trochanter of the proximal femur, while a diaphyseal AFF is a fracture that occurs in the femoral shaft distal to the subtrochanteric region and proximal to the femoral condyle [14]. Previously, these AFFs were examined as a group due to the low number of cases. However, in recent years, the differences between subtrochanteric and diaphyseal AFFs have been examined. Differences in the backgrounds of patients with each type of AFF have been reported, and the corresponding differences underlying the AFF development mechanisms in these patients have been proposed [15-20]. While it has been reported that the radius of curvature (ROC) of the femur is small in patients with diaphyseal fractures [7], no study has examined the factors inducing femoral curvature in AFF cases. Furthermore, the factors that affect the differences in the sites of AFF have not been investigated thoroughly. Therefore, this study aimed to investigate risk factors that affect diaphyseal AFFs and their etiologies.

\section{Materials and Methods}

Subjects

The medical records of 119 consecutive patients with 137 AFFs who visited our 17 institutions between June 2006 and April 2020 were retrospectively reviewed. Only female patients with diaphyseal AFFs in whom the femoral curvature angle could be examined were included because there was only 1 male patient. Fifteen patients with 15 subtrochanteric AFFs, as well as those with a history of metabolic bone diseases other than osteoporosis or malignancy, were excluded. Finally, 80 consecutive patients with 91 AFFs who exhibited at least 4 of the 5 major features, as defined by the American Society for Bone and Mineral Research Task Force, were included in the AFF group [14]. As a non-AFF control group, 110 age-matched female patients with osteoporosis were included (the osteoporosis group). Osteoporosis was diagnosed using the WHO criteria [21]. Radiographs of the entire femur were obtained to investigate the nonfracture causes of leg pain.

This study was performed in line with the ethical standards of the Helsinki Declaration of 1975, as revised in 1983. This study was also approved by the Institutional Review Board for Clinical Research at the Akita University (Approval No. 1845), and informed consent was obtained from all patients.

\section{Data Variables and Definitions}

Data were collected on the patients' age, bone resorption inhibitor usage (BPs and denosumab) or vitamin D usage before AFF onset, history of fragility fractures, complications, laboratory examination results, bone mineral density (BMD) findings, and femoral curvature measurements. The femoral curvature was measured using anteroposterior and lateral radiographs according to the method described by Yau et al. [7, 22]. The unaffected femur in patients with complete AFFs and the affected femur in patients with incomplete AFFs were examined. BMD 
was measured within 3 months after AFF diagnosis; the BMDs of the lumbar spine and of the femoral neck were measured from anteroposterior views by using a radiography system (Discovery; Hologic Inc., Marlborough, MA, USA). Furthermore, laboratory examinations were performed for determining the serum levels of bone metabolic markers and vitamin D metabolism markers, including serum albumin-adjusted calcium (Ca), inorganic phosphate, intact procollagen I N-terminal propeptide, tartrate-resistant acid phosphatase $5 \mathrm{~b}$, and $25(\mathrm{OH}) \mathrm{D}$. These parameters were measured within 2 days after AFF diagnosis. BMDs were measured within 3 months after the AFFs had been diagnosed.

It has been reported that some patients with diaphyseal AFFs exhibit a continuous and wavy thickening in the femoral endosteal region at the tensional (lateral and anterior) side (Fig. 1) [23, 24]. Even normal femoral fractures in elderly patients have exhibited this finding, though this has not yet been elucidated properly. The presence of such wavy thickening of the lateral cortex, known as a "serrated change," was also evaluated. The clinical data of the patients were compared between the AFF and the osteoporosis groups, and the factors affecting AFFs were investigated. Furthermore, the etiology of the risk factors of diaphyseal AFFs was examined.

\section{Statistical Analysis}

All continuous variables are expressed as means \pm standard deviations. The Student's $t$ test, Welch's $t$ test, and $\chi^{2}$ test were used to compare the characteristics between the groups. Multivariate logistic regression analysis was used to identify the risk factors associated with AFF and their etiologies. Probability $(p)$ values below 0.05 were considered statistically significant.

\section{Results}

In the AFF group, 11 patients had bilateral AFFs simultaneously or sequentially; $3,3,4$, and 1 patient had simultaneous bilateral insufficiency fractures, simultaneous insufficiency and complete fractures, sequential complete fractures, and a sequential complete and incomplete fracture, respectively. The remaining 69 had unilateral AFFs. Furthermore, among the 5 patients who developed AFFs sequentially, 3 were undergoing treatment for osteoporosis until the recurrence of an AFF. Sixty-nine AFFs were complete, and the remaining 22 were incomplete. Alendronate, risedronate, minodronate, or ibandronate was prescribed to 67 patients with AFFs and to 32 patients with osteoporosis. Denosumab was prescribed to 2 patients with AFFs and to 1 patient with osteoporosis.

Usage of BPs or denosumab, incidence of serrated changes in the femur, and BMDs of the proximal femurs were higher in the AFF group than in the osteoporosis group $(p<0.0001, p<0.0001$, and $p=0.0491$, respectively). The lateral and anterior femoral curvatures were

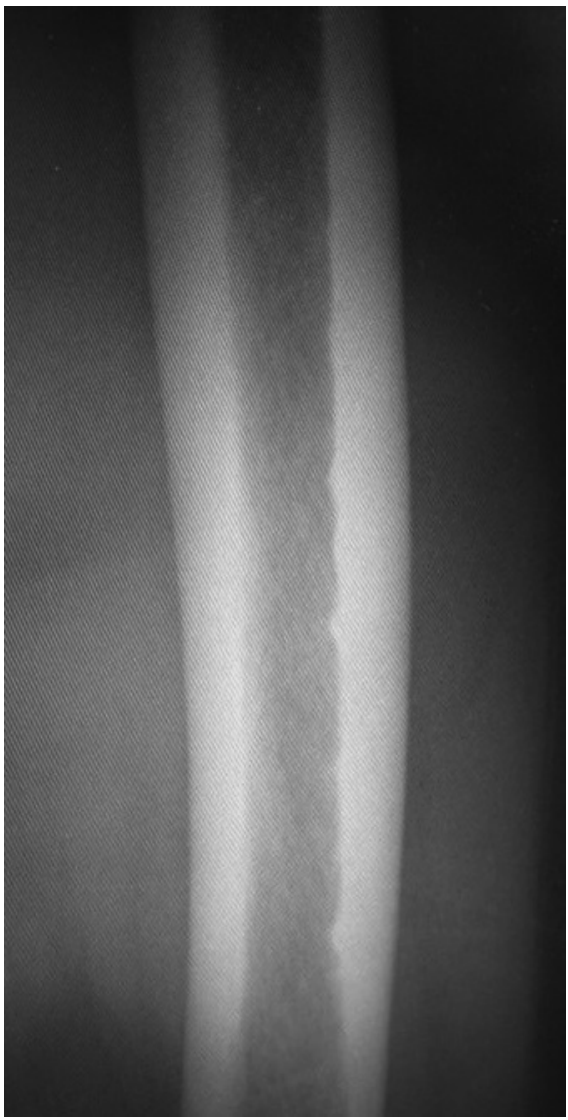

Fig. 1. Anteroposterior radiograph of the left femur presenting an example of a "serrated change." Some thickening of the femoral cortex over the outer cortical bone of the diaphysis is noted.

stronger in the AFF group ( $p<0.0001$ for both). In contrast, the incidence of previous fragility fractures was higher in the osteoporosis group than in the AFF group $(p<0.0001)$ (Table 1).

Multivariate analysis revealed serrated changes, bone resorption inhibitor usage, and lateral and anterior femoral curvatures as the risk factors for diaphyseal AFFs ( $p$ $<0.0011, p=0.0137$, and $p<0.0001$, respectively) (Table 2). All patients were divided into "curved" and "noncurved" groups using the mean and standard deviation values as the cutoff, according to our previous study [25]. The lateral curvature was $7.46^{\circ}$, and the anterior curvature was $20.55^{\circ}$. Multivariate logistic regression analysis was used to examine the factors that affected femoral curvature. Upon multivariate analysis, serrated changes, low serum $25(\mathrm{OH}) \mathrm{D}$ levels in the lateral curvature, and serrated changes only in the anterior curvature were shown to be associated with the femoral curvature $(p=0.0088$, 0.0205 , and 0.0006 , respectively) (Tables 3,4 ). Multivar- 
Table 1. Comparison of the estimated variables between the AFF group and the osteoporosis control group

\begin{tabular}{|c|c|c|c|}
\hline Patients, $N$ & 91 & 110 & \\
\hline Serrated change & $39(42.9)$ & $3(2.7)$ & $<0.0001$ \\
\hline Bone resorption inhibitor usage & $69(70.3)$ & $33(30)$ & $<0.0001$ \\
\hline Vitamin D usage & $24(26.4)$ & $16(14.5)$ & 0.0557 \\
\hline Presence of diabetes mellitus & $7(7.7)$ & $19(17.3)$ & 0.0713 \\
\hline Glucocorticoid usage & $8(8.8)$ & $4(3.6)$ & 0.2163 \\
\hline \multicolumn{4}{|l|}{ Laboratory examination } \\
\hline $\mathrm{Ca}, \mathrm{mg} / \mathrm{dL}$ & $9.25 \pm 0.47(8.4-10.8)$ & $9.15 \pm 0.33(8.2-10.1)$ & 0.1143 \\
\hline $\mathrm{iP}, \mathrm{mg} / \mathrm{dL}$ & $3.42 \pm 0.55(2.2-4.7)$ & $3.49 \pm 0.43(2.5-4.9)$ & 0.4709 \\
\hline PINP, $\mu \mathrm{g} / \mathrm{L}$ & $64.1 \pm 68.5(10.5-276)$ & $51.7 \pm 33.5(9.3-145)$ & 0.3357 \\
\hline Femoral curvature: lateral, degree & $11.6 \pm 6.1(0-25)$ & $3.6 \pm 3.3(-3.1-14.2)$ & $<0.0001$ \\
\hline Femoral curvature: anterior, degree & $18.5 \pm 7.3(3-39)$ & $10.0 \pm 2.6(4.4-17.5)$ & $<0.0001$ \\
\hline
\end{tabular}

Values are expressed as $n(\%)$ or mean \pm SD (range). AFF, atypical femoral fracture; Ca, calcium; iP, inorganic phosphorus; PINP, intact procollagen I N-terminal propeptide; TRACP5b, tartarate-resistant acid phosphatase 5b; 25(OH)D, 25-hydroxyvitamin D; BMD, bone mineral density.

Table 2. Results of the univariate and multivariate analyses of factors affecting the AFF

\begin{tabular}{|c|c|c|c|c|c|c|}
\hline Variables & OR & $95 \% \mathrm{CI}$ & $p$ value & OR & $95 \% \mathrm{CI}$ & $p$ value \\
\hline Serrated change & 26.750 & $7.896-90.623$ & $<0.0001$ & 18.543 & $3.209-106.119$ & 0.0011 \\
\hline Bone resorption inhibitor usage & 5.531 & $3.014-10.150$ & $<0.0001$ & 11.402 & $3.482-37.335$ & 0.0137 \\
\hline Vitamin D usage & 2.104 & $1.039-4.263$ & 0.0388 & 1.492 & $0.372-5.987$ & 0.5722 \\
\hline \multicolumn{7}{|l|}{ Laboratory examination } \\
\hline $\mathrm{Ca}$ & 1.941 & $0.896-4.204$ & 0.0927 & & & \\
\hline iP & 0.727 & $0.336-1.573$ & 0.4188 & & & \\
\hline PINP & 1.006 & $0.997-1.014$ & 0.1705 & & & \\
\hline TRACP-5b & 1.002 & $0.999-1.005$ & 0.1896 & & & \\
\hline \multicolumn{7}{|l|}{ Femoral curvature } \\
\hline Lateral & 1.407 & $1.280-1.547$ & $<0.0001$ & 1.271 & $1.131-1.428$ & $<0.0001$ \\
\hline Anterior & 1.454 & $1.315-1.608$ & $<0.0001$ & 1.375 & $1.213-1.559$ & $<0.0001$ \\
\hline
\end{tabular}

OR, odds ratio; 95\% CI, 95\% confidence interval; AFF, atypical femoral fracture; Ca, calcium; iP, inorganic phosphorus; PINP, intact procollagen I N-terminal propeptide; TRACP5b, tartarate-resistant acid phosphatase 5b; 25(OH)D, 25-hydroxyvitamin D; BMD, bone mineral density.

Risk Factors for Diaphyseal Atypical Femoral Fractures
Med Princ Pract 2021;30:430-436

DOI: $10.1159 / 000517484$ 
Table 3. Results of the univariate and multivariate analyses of factors affecting the lateral curvature

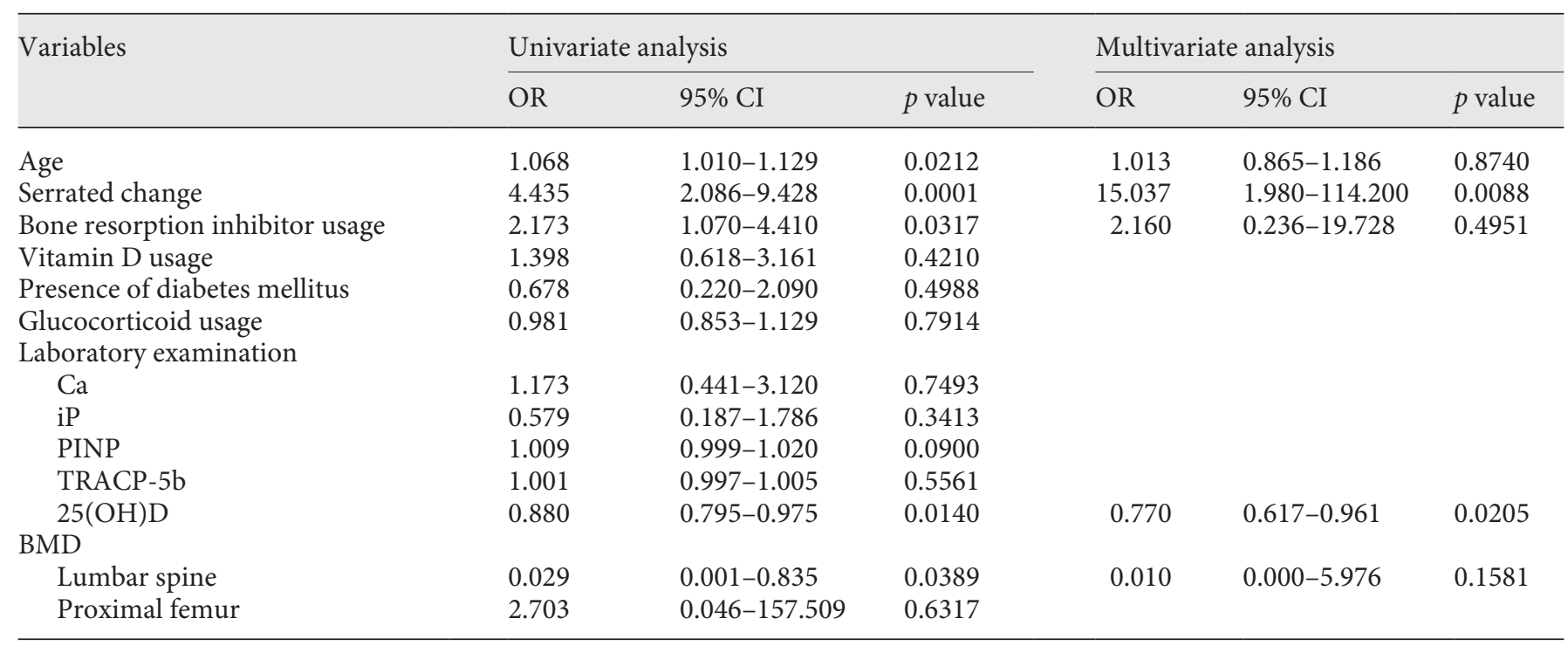

OR, odds ratio; 95\% CI, 95\% confidence interval; Ca, calcium; iP, inorganic phosphorus; PINP, intact procollagen I N-terminal propeptide; TRACP5b, tartarate-resistant acid phosphatase 5b; 25(OH)D, 25-hydroxyvitamin D; BMD, bone mineral density.

Table 4. Results of the univariate and multivariate analyses of factors affecting the anterior curvature

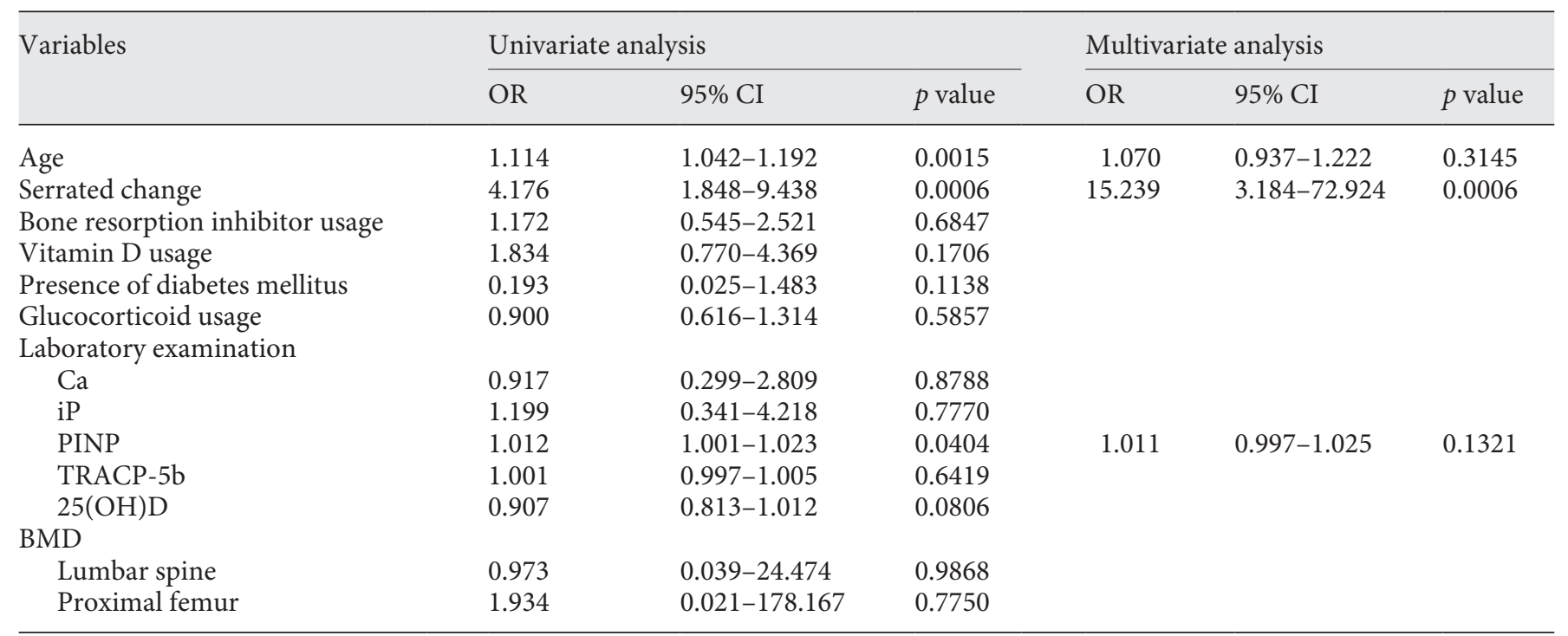

OR, odds ratio; 95\% CI, 95\% confidence interval; Ca, calcium; iP, inorganic phosphorus; PINP, intact procollagen I N-terminal propeptide; TRACP5b, tartarate-resistant acid phosphatase 5b; 25(OH)D, 25-hydroxyvitamin D; BMD, bone mineral density.

iate analyses revealed high serum Ca levels, strong femoral curvatures in the lateral dimension, and strong femoral curvatures in the anterior dimension as the predictors of serrated changes ( $p=0.0146,0.0022$, and 0.0098 , respectively) (Table 5).

\section{Discussion}

The differences between diaphyseal and subtrochanteric AFFs have been examined in several studies. It has been reported that compared to subtrochanteric AFFs, 
Table 5. Results of the univariate and multivariate analyses of factors affecting the serrated change

\begin{tabular}{|c|c|c|c|c|c|c|}
\hline Variables & OR & $95 \% \mathrm{CI}$ & $p$ value & OR & $95 \% \mathrm{CI}$ & $p$ value \\
\hline Bone resorption inhibitor usage & 1.571 & $0.791-3.120$ & 0.1971 & & & \\
\hline Vitamin D usage & 1.344 & $0.596-3.032$ & 0.4766 & & & \\
\hline Presence of diabetes mellitus & 0.281 & $0.064-1.242$ & 0.0941 & & & \\
\hline $\mathrm{Ca}$ & 3.295 & $1.219-8.411$ & 0.0126 & 3.577 & $1.286-9.946$ & 0.0146 \\
\hline iP & 1.174 & $0.493-2.791$ & 0.7174 & & & \\
\hline PINP & 1.005 & $0.995-1.015$ & 0.3376 & & & \\
\hline TRACP-5b & 1.002 & $0.999-1.006$ & 0.1845 & & & \\
\hline $25(\mathrm{OH}) \mathrm{D}$ & 0.988 & $0.937-1.043$ & 0.6693 & & & \\
\hline Lateral & 1.181 & $1.112-1.256$ & $<0.0001$ & 1.144 & $1.049-1.246$ & 0.0022 \\
\hline Anterior & 1.126 & $1.068-1.186$ & $<0.0001$ & 1.114 & $1.026-1.209$ & 0.0098 \\
\hline
\end{tabular}

OR, odds ratio; 95\% CI, 95\% confidence interval; Ca, calcium; iP, inorganic phosphorus; PINP, intact procollagen I N-terminal propeptide; TRACP5b, tartarate-resistant acid phosphatase 5b; 25(OH)D, 25-hydroxyvitamin D; BMD, bone mineral density.

diaphyseal AFFs are associated with an older age, lower $\mathrm{BMD}$, and more excessive femoral curvature [15-20]. Oh et al. [17] examined the mechanical load on femurs with and without the femoral curvature. They found that when the femoral curvature is large, the load is mostly applied to the outside of the femoral shaft, whereas when the curvature is small, the load is applied within the vicinity of the femoral trochanter; their study showed that the degree of curvature greatly influenced the site of AFF [17]. In our study, the ROC of the femur was found to be small in diaphyseal AFFs, and these AFFs were determined to have been greatly influenced by the femoral curvature.

While various studies have examined the relationship between the femoral curvature and AFFs, few studies have investigated the etiology of femoral curvatures. Our previous study, which included only older women with osteoporosis, showed that an older age and low serum $25(\mathrm{OH}) \mathrm{D}$ levels affected the femoral curvature [25]; the present study also showed that a low serum $25(\mathrm{OH}) \mathrm{D} \mathrm{lev-}$ el affected the femoral curvature. Furthermore, our previous study showed that low serum $25(\mathrm{OH}) \mathrm{D}$ levels affected both the lateral and anterior curvatures; however, the current study indicated that low serum $25(\mathrm{OH}) \mathrm{D}$ levels were only associated with the lateral curvature. The reason for the significant association between the serum
$25(\mathrm{OH}) \mathrm{D}$ levels and the lateral bow of the femur, but not the anterior bow, in patients with AFF remains unclear.

Our analysis revealed that a serrated change in the femur is a risk factor for the development of diaphyseal AFFs. Serrated changes are occasionally identified radiographically, but no studies have examined these findings; therefore, their details remain unclear. Our findings revealed that the femoral curvature is associated with the development of a serrated change; however, the mechanism underlying the occurrence of this factor remains unclear. Furthermore, a high serum Ca level has also been identified as a risk factor for serrated changes, but the mechanism underlying this remains unclear. Further examination, including histological evaluation, is necessary to evaluate serrated changes in detail.

This is the first study to perform a detailed analysis of the risk factors for diaphyseal AFFs using a large number of cases ( $>90$ cases). However, our study has several limitations. Only patients with a diaphyseal AFF and osteoporosis were compared, and patients with subtrochanteric AFFs were excluded due to their small numbers. It is important to clarify the differences between the risk factors for diaphyseal AFFs and those for subtrochanteric AFFs; although AFFs are relatively rare, more subtrochanteric AFF cases will have to be analyzed in the future to clarify these differences. 


\section{Conclusions}

To our knowledge, this study is the first to investigate factors that affect the occurrence of diaphyseal AFFs. The risk factors for diaphyseal AFFs were the use of bone resorption inhibitors, a small ROC of the femur, and serrated changes in the femur. Furthermore, a low serum $25(\mathrm{OH}) \mathrm{D}$ level was a risk factor for lateral curvature, and a high serum Ca level was a risk factor for serrated changes; these factors may influence each other. When treating patients with osteoporosis, diaphyseal AFFs should be suspected in those with these risk factors.

\section{Statement of Ethics}

This study was approved by the Institutional Review Board for Clinical Research at the Akita University (Approval No. 1845). All study participants provided informed consent.

\section{Conflict of Interest Statement}

The authors have no conflicts of interest to declare.

\section{Funding Sources}

No funding was received for this work.

\section{Author Contributions}

All authors were involved in the planning and execution of this study. Tsuchie H., Nozaka K., Saito K., Kinoshita H., Kobayashi M., Suzuki N., Aizawa T., Abe H., Maekawa S., Tomite T., Ono Y., Ouchi K., Shibata N., Nagahata I., Takeshima M., Akagawa M., Yuasa Y., and Sato C.: collection of clinical data. Tsuchie H.: analysis of raw data and drafting of the manuscript. Miyakoshi N., Kasukawa Y., and Shimada Y.: review of the manuscript.

\section{References}

1 Bamias A, Terpos E, Dimopoulos MA. Avascular osteonecrosis of the jaw as a side effect of bisphosphonate treatment. Onkologie. 2010; 33(6):288-9.

2 Odvina CV, Zerwekh JE, Rao DS, Maalouf N, Gottschalk FA, Pak CY. Severely suppressed bone turnover: a potential complication of alendronate therapy. J Clin Endocrinol Metab. 2005 Mar;90(3):1294-301.

3 Schilcher J, Michaëlsson K, Aspenberg P. Bisphosphonate use and atypical fractures of the femoral shaft. N Engl J Med. 2011 May 5; 364(18):1728-37.

4 Feldstein AC, Black D, Perrin N, Rosales AG, Friess D, Boardman D, et al. Incidence and demography of femur fractures with and without atypical features. J Bone Miner Res. 2012 May; 27(5):977-86.

5 Meier RP, Perneger TV, Stern R, Rizzoli R, Peter RE. Increasing occurrence of atypical femoral fractures associated with bisphosphonate use. Arch Intern Med. 2012 Jun 25;172(12):930-6.

6 Melton LJ III, Chrischilles EA, Cooper C, Lane AW, Riggs BL. Perspective: how many women have osteoporosis? J Bone Miner Res. 1992 Sep; 7(9):1005-10.

7 Sasaki S, Miyakoshi N, Hongo M, Kasukawa Y, Shimada Y. Low-energy diaphyseal femoral fractures associated with bisphosphonate use and severe curved femur: a case series. J Bone Miner Metab. 2012 Sep;30(5):561-7.

8 Girgis CM, Sher D, Seibel MJ. Atypical femoral fractures and bisphosphonate use. N Engl J Med. 2010 May 13;362(19):1848-9.

9 Rasmussen NH, Dal J, de Vries F, van den Bergh JP, Jensen MH, Vestergaard P. Diabetes and fractures: new evidence of atypical femoral fractures? Osteoporos Int. 2020 Mar;31(3): $447-55$.
10 Lee YK, Kim TY, Ha YC, Song SH, Kim JW, Shon HC, et al. Atypical subtrochanteric fractures in Korean hip fracture study. Osteoporos Int. 2017 Oct;28(10):2853-8.

11 Gustafsson A, Schilcher J, Grassi L, Aspenberg $\mathrm{P}$, Isaksson $\mathrm{H}$. Strains caused by daily loading might be responsible for delayed healing of an incomplete atypical femoral fracture. Bone. 2016 Jul;88:125-30.

12 Schilcher J, Koeppen V, Aspenberg P, Michaëlsson K. Risk of atypical femoral fracture during and after bisphosphonate use. Acta Orthop. 2015 Feb;86(1):100-7.

13 Koh JH, Myong JP, Yoo J, Lim YW, Lee J, Kwok SK, et al. Predisposing factors associated with atypical femur fracture among postmenopausal Korean women receiving bisphosphonate therapy: 8 years' experience in a single center. Osteoporos Int. 2017 Nov;28(11):3251-9.

14 Yoo H, Cho Y, Park Y, Ha S. Lateral femoral bowing and the location of atypical femoral fractures. Hip Pelvis. 2017 Jun;29(2):127-32.

15 Kim JW, Kim JJ, Byun YS, Shon OJ, Oh HK, Park KC, et al. Factors affecting fracture location in atypical femoral fractures: a cross-sectional study with 147 patients. Injury. 2017 Jul; 48(7):1570-4.

16 Oh Y, Fujita K, Wakabayashi Y, Kurosa Y, Okawa A. Location of atypical femoral fracture can be determined by tensile stress distribution influenced by femoral bowing and neck-shaft angle: a CT-based nonlinear finite element analysis model for the assessment of femoral shaft loading stress. Injury. 2017 Dec;48(12):273643.

17 Cho YJ, Kang KC, Chun YS, Rhyu KH, Kim SJ, Jang TS. Critical differences between subtrochanteric and diaphyseal atypical femoral fractures: analyses of 51 cases at a single institution in Korean population. Arch Osteoporos. 2018 May 3;13(1):53

18 Park YC, Yoon SP, Yang KH. Localization of atypical femoral fracture on straight and bowed femurs. J Bone Metab. 2019 May;26(2):123-31.

19 Tano A, Oh Y, Fukushima K, Kurosa Y, Wakabayashi Y, Fujita K, et al. Potential bone fragility of mid-shaft atypical femoral fracture: biomechanical analysis by a CT-based nonlinear finite element method. Injury. 2019 Nov; 50(11):1876-82.

20 Shane E, Burr D, Abrahamsen B, Adler RA, Brown TD, Cheung AM, et al. Atypical subtrochanteric and diaphyseal femoral fractures: second report of a task force of the American society for bone and mineral research. J Bone Miner Res. 2014 Jan;29(1):1-23.

21 WHO Scientific Group. Prevention and management of osteoporosis. World Health Organ Tech Rep Ser. 2003;921:1-164.

22 Yau WP, Chiu KY, Tang WM, Ng TP. Coronal bowing of the femur and tibia in Chinese: its incidence and effects on total knee arthroplasty planning. J Orthop Surg. 2007 Apr;15(1): 32-6.

23 Tsuchie H, Miyakoshi N, Nishi T, Abe H, Segawa T, Shimada Y. A case of incomplete atypical femoral fracture with histomorphometrical evidence of osteomalacia. Acta Med Okayama. 2015;69(1):59-63.

24 Tsuchie H, Miyakoshi N, Nishi T, Abe H, Segawa T, Shimada Y. Combined effect of a locking plate and teriparatide for incomplete atypical femoral fracture: two case reports of curved femurs. Case Rep Orthop. 2015;2015:213614.

25 Tsuchie H, Miyakoshi N, Kasukawa Y, Senma S, Narita Y, Miyamoto S, et al. Factors related to curved femur in elderly Japanese women. Ups J Med Sci. 2016 Aug;121(3):170-3. 